\title{
Communication
}

\section{Strain distribution in wrinkled hBN films}

\author{
K. Bera ${ }^{\mathrm{a}}$, D. Chugh ${ }^{\mathrm{b}}$, Atanu Patra ${ }^{\mathrm{c}}$, H. Hoe Tan ${ }^{\mathrm{b}, \mathrm{d}}$, C. Jagadish ${ }^{\mathrm{b}, \mathrm{d}}$, Anushree Roy ${ }^{\mathrm{c}, * *}$ \\ ${ }^{a}$ School of Nanoscience and Technology, Indian Institute of Technology Kharagpur, 721302, India \\ ${ }^{\mathrm{b}}$ Department of Electronic Materials Engineering, Research School of Physics, The Australian National University, Canberra, ACT, 2601, Australia \\ ${ }^{\mathrm{c}}$ Department of Physics, Indian Institute of Technology Kharagpur, 721302, India \\ ${ }^{\mathrm{d}}$ Australian Research Council Centre of Excellence for Transformative Meta-Optical Systems, The Australian National University, Canberra, ACT, 2601, Australia
}

\section{A B S T R A C T}

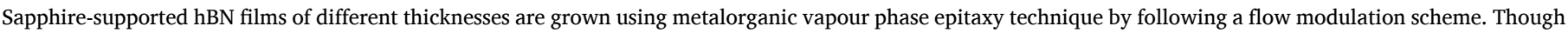

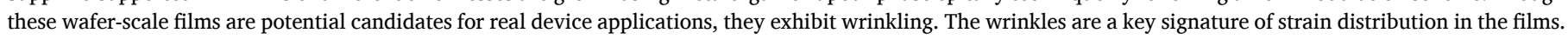

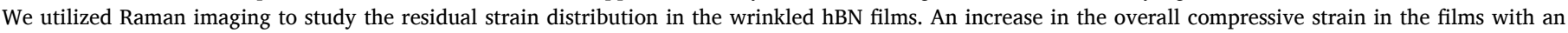

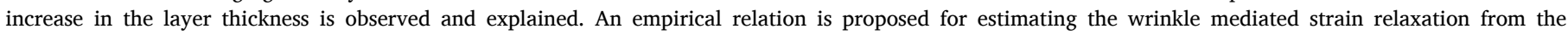
morphology of the films. Furthermore, we show that the residual strain can be partially released by the delamination of the films.

\section{Introduction}

Recently, thin layers of hexagonal boron nitride (hBN) have attracted remarkable attention for their unique physical properties [1]. They are often used as buffer layers in the development of a wide range of advanced electronic and optoelectronic 2D material based devices [2,3]. For example, in the fabrication of capacitors based hBN/graphene/hBN heterostructures, a thin layer of hBN serves both as an insulating as well as an encapsulating layer [4,5].

Unique characteristics of the hBN layers, obtained from mechanical exfoliation of bulk crystals, are well-documented. However, the exfoliated films find limitations in many applications due to the size restriction. In recent studies, the possibility of large scale growth of hBN films on lattice mismatched substrates has been discussed [6]. Though these dielectric films, grown on a relatively large area, are potential candidates as dielectric layers for 2D material based field-effect devices, wrinkling has been observed in as-grown layers.

The physics of wrinkling is complex. It depends on various parameters, like elastic properties and the thicknesses of both film and substrate, substrate anchorage, etc. [7-9]. Quite a few reports in the literature have discussed the characteristics of wrinkles in a similar system, like graphene [9-12]. During the growth process, strain in the films develops due to the opposite polarity of the thermal expansion coefficients of the film and the substrate [9]. Wrinkling partially relaxes the strain in the films. For example, the relaxation of lateral strain, developed at the time of cooling of CVD (chemical vapour deposition) grown graphene on solid metallic substrate, results in localized or linear wrinkle network in the films $[10,11]$. Using shear-lag analysis, it has been shown that in wrinkled graphene island, the residual strain vanishes at the boundary and highest at the centre of the wrinkled domain [12]. The characteristics of wrinkles of a substrate supported hBN film are expected to be very similar to those of graphene, as both have negative thermal expansion coefficient and $\mathrm{sp}^{2}$ network atoms in a hexagonal arrangement. In the literature, the wrinkles in the as-grown hBN films on Cu-foil [13] and sapphire [6] are observed. The above studies motivated us to investigate and quantify the spatial non-uniform strain distribution in as-grown hBN films and, more importantly, to prescribe a possible way to remove it or to limit it to an acceptable value.

Raman spectroscopy is an indispensable technique to probe the lattice strain in 2D materials [14-18]. In this article, we study the residual strain distribution in hBN films of different thicknesses, grown on sapphire substrates by metal organic vapour phase epitaxy (MOVPE) technique, using Raman mapping. We observe an overall compressive strain in the films from the mapping of the Raman shift of the $E_{2 g}^{\text {high }}$ phonon mode of hBN. An empirical relation is proposed for estimating the residual strain from the morphology of the film. The mapping of the Raman line-width reveals an improvement in the crystallinity of the film with thickness. Furthermore, we demonstrate that the compressive strain in the films can be partially removed by delamination.

\section{Experimental}

hBN films were deposited on commercially available $2^{\prime \prime}$ sapphire substrates using flow modulation MOVPE growth technique in an

\footnotetext{
* Corresponding author.

E-mail address: anushree@phy.iitkgp.ernet.in (A. Roy).
} 

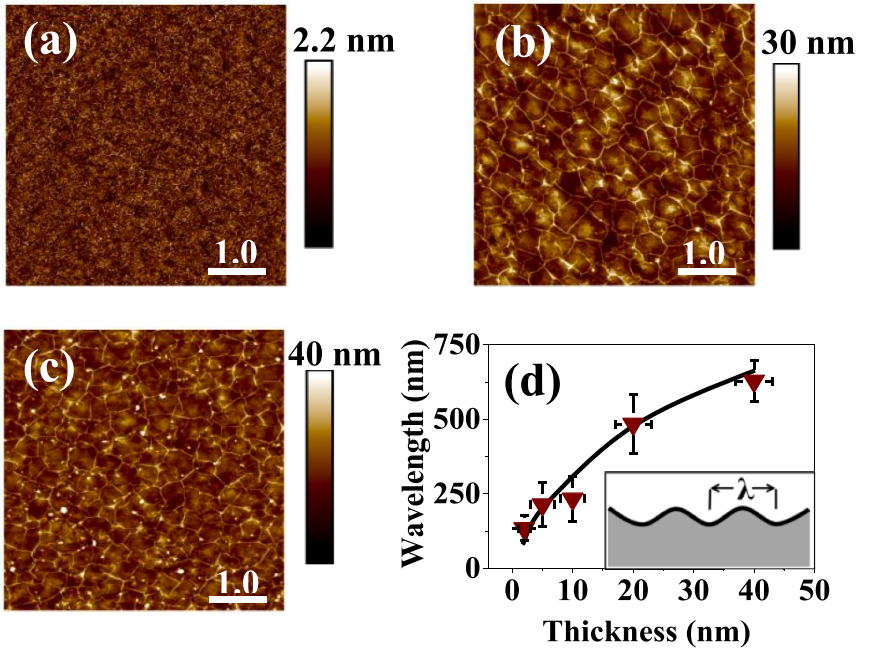

Fig. 1. Surface morphology of (a) $2 \mathrm{~nm}$, (b) $20 \mathrm{~nm}$ and (c) $40 \mathrm{~nm}$ thick hBN films on sapphire substrates at room temperature. (d) The variation of average value of the wrinkle wavelength, $\lambda$, with the film thickness, $h$. The solid line is a guide to the eyes. Inset of (d) schematically defines the wrinkle wavelength $\lambda$.

Aixtron MOVPE close-coupled showerhead (CCS) reactor. Triethylboron (TEB) and ammonia $\left(\mathrm{NH}_{3}\right)$ were used as boron and nitrogen precursors, respectively. The substrate temperature was kept fixed at $1350{ }^{\circ} \mathrm{C}$ during the growth process. The thickness of the films was controlled by varying the growth duration. Experiments were also carried out on hBN films transferred on $\mathrm{SiO}_{2} / \mathrm{Si}$ substrates. In the presence of de-ionized water, the MOVPE grown hBN films self-delaminate from the substrate. The details of the growth and film transfer processes are available in Ref. [6].

A tapping mode AFM (SCANASYSTAIR, MultiMode 8, Bruker) was used to observe the surface morphology and measure the film thickness. For micro-Raman spectroscopy, a triple grating spectrometer (T64000, Horiba, France) was used in the subtractive mode. The unit was equipped with a Peltier cooled CCD detector (Synapse, Horiba, France), a water-cooled Argon-Krypton ion laser (Innova 70C Spectrum, Coherent, USA) and an optical microscope (BX41, Olympus, Japan). To collect the backscattered radiation, $100 \times$ (numerical aperture 0.90 ) objective lens was used. Spectra were recorded using $514 \mathrm{~nm}$ laser excitation wavelength with $2.0 \mathrm{~mW}$ power on the sample. Point by point Raman mapping of the films over $1.98 \mu \mathrm{m} \times 0.88 \mu \mathrm{m}$ with a step size of 0.220 $\mu \mathrm{m}$ on films was carried out using a computer controlled motorized $\mathrm{XY}$ stage.

\section{Results and discussion}

Fig. 1(a)-(c) exhibit characteristic AFM images of some of the films grown for different time durations. The colour scale in the right of each panel marks the nanoscale topographical information of the surface of the films. The formation of wrinkles throughout each film is observed. From the height line profiles (not shown), we have measured the thickness $(h)$ of the films. In this article, we study the films of thicknesses, $2 \pm 1 \mathrm{~nm}, 5 \pm 2 \mathrm{~nm}, 10 \pm 2 \mathrm{~nm}, 20 \pm 3 \mathrm{~nm}$, and $40 \pm 3 \mathrm{~nm}$.

To describe the kinetics of wrinkles, two parameters, wrinkle wavelength, $\lambda$ (see the inset of Fig. 1(d)), and wrinkle height, $h$, (same as amplitude) $[7,8]$, are often used. The wrinkled domains in the films are non-uniform in shape (see Fig. 1(a)-(c)). Thus, to estimate $\lambda$, we followed the procedure described in Ref. [13] by measuring the average length of two crossed lines across each domain (see Fig. S1). Nearly 80 domains from several image frames of each film were analysed. The variation of the mean values of $\lambda$ with $h$, as measured from the AFM images, is shown by symbols in Fig. 1(d). The error bars in $\lambda$ are the standard deviations of the values of the measured parameter. The solid line is a guide to the eyes to follow the $\lambda$ vs. $h$ plot. We find that the characteristic of the wrinkles varies significantly with the film thickness.

Raman spectra of the $\mathrm{E}_{2 \mathrm{~g}}^{\text {high }}$ phonon mode of the films are shown by + symbols in Fig. 2(a). The topmost spectrum is that of bulk hBN. The red dashed line marks the blue shift in the spectra of the films. As mentioned earlier, wrinkling may leave a residual strain in the films. The wrinkled surface topography in Fig. 1 and hence, the possibility of spatial nonuniform residual strain in the films indicates that the structural characteristics may not be uniform over the film area. Thus, the single point Raman measurements (shown in Fig. 2(a)) may not reflect the true consequence of wrinkling in the films. To address this issue, we carry out Raman mapping, over a reasonably large area encompassing multiple wrinkled domains. The Raman image provides an overall (mean) characteristic of the entire film. In this imaging technique, spectra are collected at each point of a raster-scanned sample. False colour images, which are based on the spectral parameters of the characteristic Raman mode of the films, are then generated.

The map of Raman line-width, revealing the variation is crystallinity

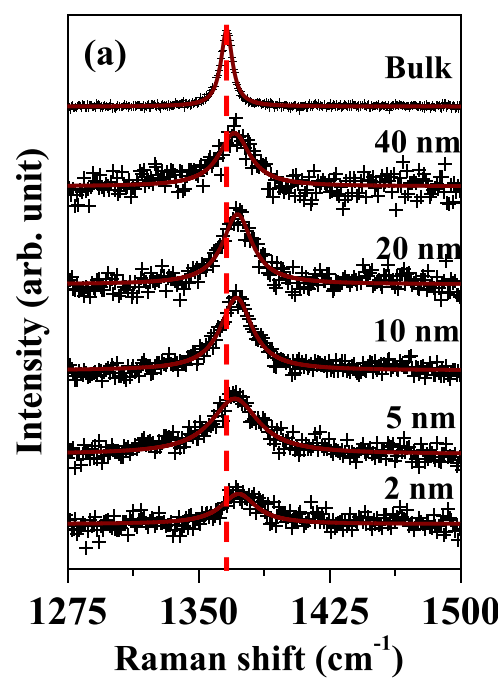

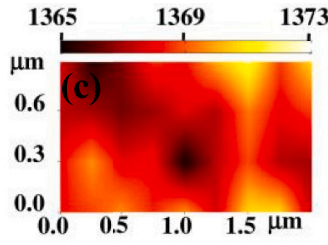
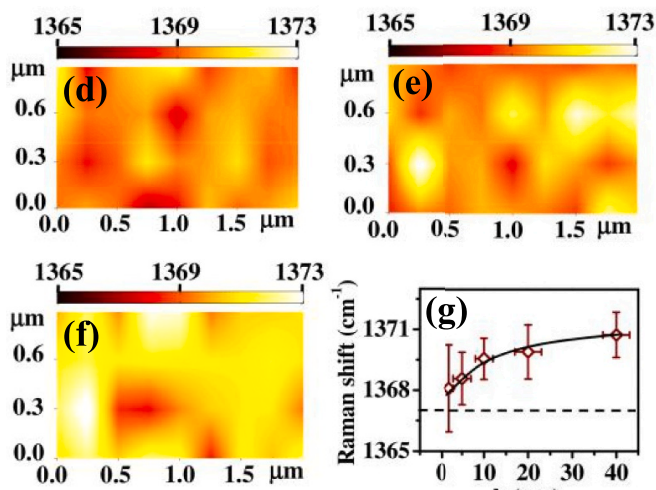

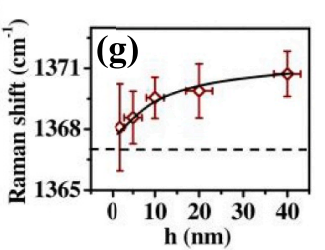

Fig. 2. (a) Raman spectra of hBN films of different thicknesses, including bulk hBN for reference. The net fitted spectrum for each sample, obtained by fitting the data points (+symbols) using a Lorentzian function is shown by the solid line. The red dashed line marks the shift in the spectra of the films. Mapping of the Raman shift of the $\mathrm{E}_{2 \mathrm{~g}}^{\text {high }}$ mode for (b) $2 \pm 1 \mathrm{~nm}$ (c) $5 \pm 2 \mathrm{~nm}$, (d) $10 \pm 2 \mathrm{~nm}$, (e) $20 \pm 3 \mathrm{~nm}$ and (f) $40 \pm 3 \mathrm{~nm}$ thick hBN films. The scale bars are in $\mathrm{cm}^{-1}$. (g) Variation of the mean value of Raman shift of the $E_{2 g}^{\text {high }}$ mode with film thickness, as obtained by analysing each spectrum of the mapped area. The vertical error bar to each data point is the standard deviation of all values obtained by analysing all spectra in the corresponding image frame. The solid line is a guide to the eyes. (For interpretation of the references to colour in this figure legend, the reader is referred to the Web version of this article.) 
in the films, is discussed in the supplementary information (S2). Fig. 2 (b)-(f) present characteristic map of Raman shift of the $\mathrm{E}_{2 \mathrm{~g}}^{\text {high }}$ vibrational mode of hBN films of thicknesses $2 \pm 1 \mathrm{~nm}, 5 \pm 2 \mathrm{~nm}, 10 \pm 2 \mathrm{~nm}, 20 \pm 3$ $\mathrm{nm}$, and $40 \pm 3 \mathrm{~nm}$, respectively. The colour bar on the top of each panel exhibits the scale in $\mathrm{cm}^{-1}$. For a better comparison of the change in this parameter with the thickness of the films, the colour scale bars are kept the same for all images. Raman shift exhibits non-uniformity over the scanned area. The saturation in colour in some of the images (say for Fig. 2(e) and (f)) indicates that the ranges of the measured parameters are greater than the range used in the scale bar. The far field diffraction of laser light limits us to relate the observation to the domain characteristics of these films. However, the Raman images render their overall structural properties.

Fig. 2(g) plots the variation of the mean value of Raman shift over the scanned area with film thickness and vertical error bars are the standard deviation. The measured Raman wavenumber $\left(1367 \mathrm{~cm}^{-1}\right)$ of the $\mathrm{E}_{2 \mathrm{~g}}^{\text {high }}$ phonon mode of bulk hBN is shown by a dashed line in Fig. 2(g). Due to non-resonant experimental condition, and low laser power used in our measurements, the signal to noise ratio of the hBN spectrum was low. This resulted in large standard deviation of the data points, especially for thinner films. Nonetheless, we observe a blue shift in Raman wavenumber of the films from its bulk value. It is also interesting to note there is an increasing trend in Raman shift with the increase in film thickness. For free-standing exfoliated films, the frequency of the intralayer $E_{2 g}^{\text {high }}$ vibrational mode is independent of the number of layers $[16,17]$. Thus, the observed increase of the same mode in Fig. 2(g) for hBN/sapphire contradicts the behaviour of the suspended films.

Below we argue several possibilities, which can result in a shift of the phonon mode of a film. (i) Laser heating can give rise to a red shift in the phonon mode [18]. Measurements on a single point were carried out with an acquisition time of hours. We did not find any shift in the spectrum. Furthermore, heat dissipation via the substrate is expected to be higher in the case of thinner films than in the thicker ones. In addition, the thermal conductivity of hBN films increases with the decrease in film thickness [18]. Thus, one expects a higher rise in temperature in thicker films and hence a red shift in their phonon wavenumber than in the thinner layers, which contradicts the observation in Fig. 2(g). Thus, we strongly believe that the increase in temperature due to absorbed laser power by the films is not the origin of the observed blue shift with the increase in the thickness of the films. (ii) We rule out the role of substrate doping in the shift of the phonon mode, as hBN is a high band gap insulator [19]. (iii) The strain in a film modifies the deformation potential and optical phonon interaction, and hence, results in a shift of the phonon frequency [20]. Elimination of the possibilities (i) and (ii) mentioned above, strongly suggests that the observed blue shift of Raman frequency in Fig. 2(g) originates from the residual strain in the wrinkled films.

By solving the dynamical equation of the phonon mode under strain in a $2 \mathrm{D}$ hexagonal lattice, one obtains the shift in phonon frequency as (to the first order) [15].

$\Delta \omega_{ \pm}=\Delta \omega^{h} \pm \frac{1}{2} \Delta \omega^{s}=-\omega_{u s} \gamma\left(\varepsilon_{l l}+\varepsilon_{t t}\right) \pm \frac{1}{2} \beta \omega_{u s}\left(\varepsilon_{l l}-\varepsilon_{t t}\right)$.

$\Delta \omega^{h}$ and $\Delta \omega^{s}$ are shifts in phonon frequency due to hydrostatic and shear strain in the films, respectively. $\gamma$ is the Grüneisen parameter. $\beta$ corresponds to the shear deformation potential in the lattice. $\omega_{u s}$ is the $E_{2 g}^{\text {high }}$ mode frequency of the unstrained bulk hBN. $\varepsilon_{l l}$ and $\varepsilon_{t t}$ are strain tensor components, $l$ being parallel to the direction of strain, and $t$ is perpendicular to the same. Symmetry breaking $\left(\varepsilon_{l l} \neq \varepsilon_{t t}\right)$ of the lattice can give rise to a splitting of the $\mathrm{E}_{2 \mathrm{~g}}$ mode. Under biaxial strain with $\varepsilon_{l l}=\varepsilon_{t t}$, the $\mathrm{E}_{2 \mathrm{~g}}$ mode does not show any splitting due to the cancellation of the shear deformation. As we did not observe any splitting of the $E_{2 g}$ mode even when measured at 150 and $78 \mathrm{~K}$ (not shown), we believe that only a biaxial strain with $\varepsilon_{l l} \approx \varepsilon_{t t}$ is present in the hBN films. In Eqn. (1), considering only the hydrostatic component of the net strain tensor, we
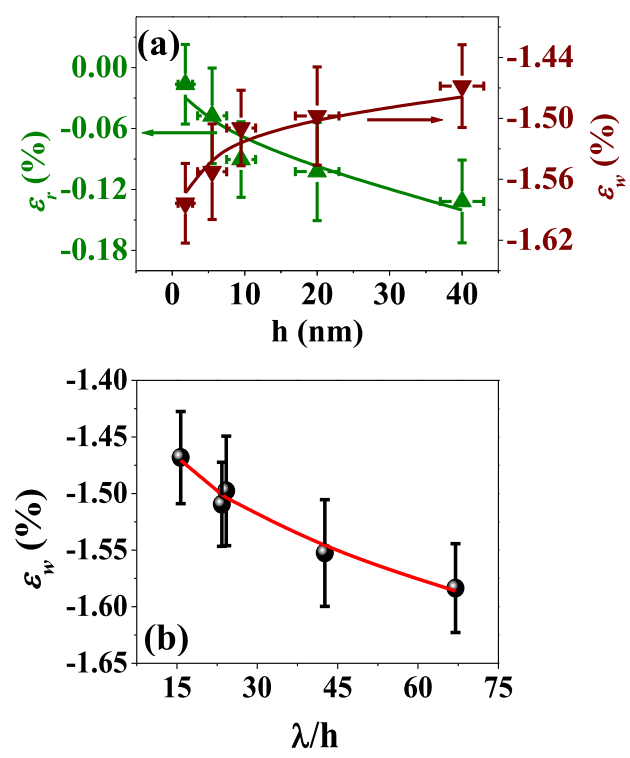

Fig. 3. (a) Variation of residual strain $\left(\varepsilon_{r}\right)$ (left scale) and strain relaxation $\left(\varepsilon_{w}\right)$ by wrinkling (right scale) with film thickness. (b) Change in $\varepsilon_{w}$ with $\lambda / h$. The solid line is the fit the data point using the empirical relation, discussed in the text.

find $[14,15]$.

$\varepsilon_{l l}=\varepsilon_{t t}=\varepsilon_{r}=-\Delta \omega / 2 \gamma \omega_{u s}$

A positive value of $\Delta \omega$ (blue shift in Raman wavenumber) renders a compressive strain, while for a tensile strain $\Delta \omega$ is negative. We find the value of $\gamma$ for different film thicknesses from Ref. 15. The estimated values of the mean residual strain, $\varepsilon_{r}$, of the films of different thicknesses, as obtained by subtracting the mean value of the Raman shift of films from Raman shift of bulk hBN in Fig. 2(g), are plotted in Fig. 3(a) by green up-pointing triangle symbols. We find higher compressive mean residual strain in the thicker films.

In the reaction chamber, after the growth is stopped and the substrate is allowed to cool, while the sapphire tries to contract, the hBN film has a tendency to expand. Once the strain in the film is beyond a critical value, the effect is manifested by the bending (local decohesion) of the film from the substrate (wrinkling) [8]. We argue that the relaxation of strain by bending is more effective for fewer layers, as with the increase in the number of layers the interplanar van der Waals interaction also plays a role and hinders strain relaxation by bending (wrinkling).

To estimate the strain relaxed by wrinkling, first we find the expected strain in the films (prior to wrinkling) due to the difference in the elastic properties of the film and the substrate. For substrates with a positive thermal expansion coefficient, it has been shown that the evolution of strain in hBN films follows a linear relation [21,22] according to following the relation $\varepsilon_{t h}=\int_{T_{0}}^{T_{f}}\left(\alpha_{s}-\alpha_{f}\right) d T$. This expression is expected to be valid for small strains in smooth films [21]. In the present case of the MOVPE grown films, the initial $\left(T_{0}\right)$ and final $\left(T_{\mathrm{f}}\right)$ temperatures are 1350 and $27^{\circ} \mathrm{C}$, respectively. $\alpha_{f}$ and $\alpha_{s}$ are the linear thermal expansion coefficients of the hBN film and sapphire substrate, respectively. Using $\alpha_{f}=-2.96 \times 10^{-6}+1.91 \mathrm{~T} \times 10^{-9} /{ }^{\circ} \mathrm{C}[22]$ and $\alpha_{s}=4.5 \times 10^{-6}+6.2(\mathrm{~T}+273) \times 10^{-9} /{ }^{\circ} \mathrm{C}$ [23], the calculated biaxial compressive strain $\left(\varepsilon_{t h}\right)$ is typically $1.6 \%$ before wrinkling occurs in the films. Although wrinkling reduces a part of this compressive strain, the film may not be strain free, as revealed from the residual strain measurements in Fig. 3(a). The expected overall strain relaxation $\left(\varepsilon_{w}\right)$ in the films by wrinkling is then estimated by considering $\varepsilon_{w}=\varepsilon_{t h}{ }^{-} \varepsilon_{r}$ and plotted in Fig. 3(a) by red down-pointing triangle symbols. 

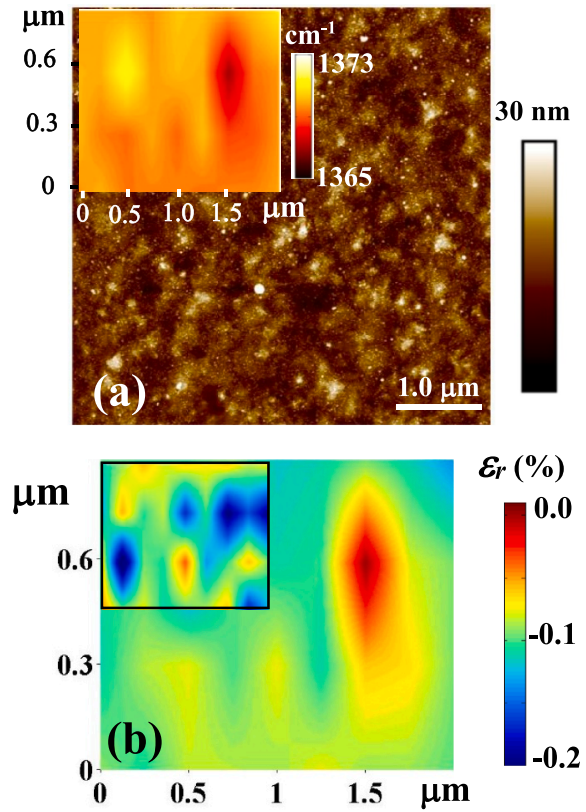

Fig. 4. (a) AFM image of the $20 \mathrm{~nm}$ thick delaminated film. Inset shows the mapping of the Raman shift of the $E_{2 g}^{\text {high }}$ mode of the delaminated film. (b) Mapping of residual strain for the $20 \mathrm{~nm}$ delaminated film. The inset shows the same mapping for the substrate-supported film using the same scale bar on the right.

Using the finite element theory it has been demonstrated that the strain in a substrate supported wrinkled film follows a power law behaviour with the wrinkle wavelength $(\lambda)$ and thickness $(h)$ of the film $[7,8,24]$. For example, for a viscous substrate, the strain in the film is proportional to the square of the ratio of $h$ and $\lambda$ [8]. To best of our knowledge, such a study is still unavailable in the literature for hBN films grown on a substrate with opposite polarity of thermal expansion coefficient. By fitting $\varepsilon_{w}$ in Fig. 3(b) with a polynomial function of $(\lambda / h)$ we propose an empirical relation

$\varepsilon_{w}=-0.3\left(\frac{\lambda}{h}\right)^{0.05}$

for an estimation of the strain relaxed by wrinkling from the surface morphology of the hBN films on sapphire.

Furthermore, we investigated the residual strain distribution in the delaminated films. Fig. 4(a) shows a characteristic AFM image of the delaminated $20 \mathrm{~nm}$ film. By comparing the AFM image of the same film on a sapphire substrate (as-grown) in Fig. 1(b), we note that the delaminated film is free of wrinkles. A similar change in surface morphology is observed in AFM images of the delaminated $40 \mathrm{~nm}$ thick $\mathrm{hBN}$ film. Raman map of the $\mathrm{E}_{2 \mathrm{~g}}^{\text {high }}$ phonon mode of the $20 \mathrm{~nm}$ delaminated film is shown in the inset of Fig. 4(a). The mapping of strain in this delaminated film, using Eqn. (2), is shown in Fig. 4(b). To map the strain, we considered the difference in Raman shift at each point of the mapped area of the film (as shown in Fig. 4(a)) and bulk hBN. A similar map for substrate-supported film, as obtained by using the data in Fig. 2 (e), is shown in the inset. We find that the strain distribution is significantly less in the delaminated film. Weak van der Waals like interaction prevails between delaminated film and $\mathrm{SiO}_{2} / \mathrm{Si}$ substrate [25]. Thin hBN films have less bending moduli [26]. Thus, they easily corrugate. The uneven surface of the substrate renders a nominal strain distribution in the films. A similar observation is reported by Cullen et al, in which authors have shown that the uneven surface of $\mathrm{SiO}_{2}$ is responsible for strain in transferred exfoliated graphene layers [27].

\section{Summary}

In summary, we have probed non-uniform residual strain distribution in MOVPE grown hBN films using Raman mapping. The variation in compressive strain distribution with film thickness is discussed. An empirical relation is proposed for the estimation of overall strain relaxed by the wrinkling from the morphology of the films. The present study not only discusses the strain profile of the as-grown hBN films of varying thicknesses but also reveals a possible way to obtain strain-free hBN films for future applications.

\section{Declaration of competing interest}

The authors declare that they have no known competing financial interests or personal relationships that could have appeared to influence the work reported in this paper.

\section{CRediT authorship contribution statement}

K. Bera: Investigation, Formal analysis, Writing - original draft, Writing - review \& editing. D. Chugh: Writing - review \& editing. Atanu Patra: Formal analysis. H. Hoe Tan: Conceptualization, Writing - review \& editing. C. Jagadish: Conceptualization, Writing - review \& editing. Anushree Roy: Conceptualization, Writing - review \& editing.

\section{Acknowledgments}

The Australian authors would like thank the Australian Research Council for financial support. We acknowledge the Australian National Fabrication Facility, ACT Node for access to the epitaxial facility used in this work.

\section{Appendix A. Supplementary data}

Supplementary data to this article can be found online at https://doi. org/10.1016/j.ssc.2020.113847.

\section{References}

[1] L.H. Li, Y. Chen, Adv. Funct. Mater. 26 (2016) 2594.

[2] C.R. Dean, A.F. Young, I. Meric, C. Lee, L. Wang, S. Sorgenfrei, K. Watanabe, T. Taniguchi, P. Kim, K.L. Shepard, J. Hone, Nat. Nanotechnol. 5 (2010) 722.

[3] K. Ahmed, R. Dahal, A. Weltz, J.-Q. Lu, Y. Danon, I.B. Bhat, Appl. Phys. Lett. 109 (2016) 113501.

[4] M. Gurram, S. Omar, B.J. van Wees, 2D Mater. 5 (2018), 032004.

[5] M. Šiškins, C. Mullan, S.-K. Son, J. Yin, K. Watanabe, T. Taniguchi, D. Ghazaryan, K.S. Novoselov, A. Mishchenko, Appl. Phys. Lett. 114 (2019) 123104.

[6] D. Chugh, J. Wong-Leung, L. Li, M. Lysevych, H.H. Tan, C. Jagadish, 2D Mater. 5 (2018), 045018.

[7] E. Cerda, L. Mahadevan, Phys. Rev. Lett. 90 (2003), 074302.

[8] A.R. Shugurov, A.V. Panin, Phys. Mesomech. 13 (2010) 79.

[9] S. Deng, V. Berry, Mater. Today 19 (2016) 197.

[10] S.J. Chae, F. Günes, K.K. Kim, E.S. Kim, G.H. Han, S.M. Kim, H.J. Shin, S.M. Yoon, J.Y. Choi, M.H. Park, C.W. Yang, D. Pribat, Y.H. Lee, Adv. Mater. 21 (2009) 2328.

[11] L. Meng, Y. Su, D. Geng, G. Yu, Y. Liu, R.F. Dou, J.C. Nie, L. He, Appl. Phys. Lett. 103 (2013) 251610.

[12] Z. Li, I.A. Kinloch, R.J. Young, K.S. Novoselov, G. Anagnostopoulos, J. Parthenios, C. Galiotis, K. Papagelis, C.-Y. Lu, L. Britnell, ACS Nano 9 (2015) 3917.

[13] J. Lin, R.Y. Tay, H. Li, L. Jing, S.H. Tsang, H. Wang, M. Zhu, D.G. McCullochd, E.H. T. Teo, Nanoscale 10 (2018) 16243.

[14] C. Androulidakis, E.N. Koukaras, M. Poss, K. Papagelis, C. Galiotis, S. Tawfick, Phys. Rev. B 97 (2018), 241414 (R).

[15] T.M.G. Mohiuddin, A. Lombardo, R.R. Nair, A. Bonetti, G. Savini, R. Jalil, N. Bonini, D.M. Basko, C. Galiotis, N. Marzari, K.S. Novoselov, A.K. Geim, A. C. Ferrari, Phys. Rev. B 79 (2009) 205433.

[16] P.H. Tan, W.P. Han, W.J. Zhao, Z.H. Wu, K. Chang, H. Wang, Y.F. Wang, N. Bonini, N. Marzari, N. Pugno, G. Savini, A. Lombardo, A.C. Ferrari, Nat. Mater. 11 (2012) 294.

[17] I. Stenger, L. Schué, M. Boukhicha, B. Berini, B. Plaçais, A. Loiseau, J. Barjon, 2D Mater. 4 (2017), 031003.

[18] Q. Cai, D. Scullion, W. Gan, A. Falin, S. Zhang, K. Watanabe, T. Taniguchi, Y. Chen, E.J.G. Santos, L.H. Li, Sci. Adv. 5 (2019), eaav0129. 
[19] Q. Cai, A. Du, G. Gao, S. Mateti, B.C.C. Cowie, D. Qian, S. Zhang, Y. Lu, L. Fu, T. Taniguchi, S. Huang, Y. Chen, R.S. Ruoff, L.H. Li, Adv. Funct. Mater. 26 (2016) 8202.

[20] F. Cerdeira, C.J. Buchenauer, F.H. Pollak, M. Cardona, Phys. Rev. B 5 (1972) 580

[21] W. Pan, J. Xiao, J. Zhu, C. Yu, G. Zhang, Z. Ni, K. Watanabe, T. Taniguchi, Y. Shi, X. Wang, Sci. Rep. 2 (2012) 893.

[22] Q. Cai, D. Scullion, A. Falin, K. Watanabe, T. Taniguchi, Y. Chen, E.J.G. Santos, L. H. Li, Nanoscale 9 (2017) 3059.

[23] L.B. Freund, S. Suresh, Thin Film Materials Stress, Defect Formation and Surface Evolution, 2004.
[24] J. Zang, X. Zhao, Y. Cao, J.W. Hutchinson, J. Mech. Phys. Solid. 60 (2012) 1265.

[25] K.S. Novoselov, A. Mishchenko, A. Carvalho, A.H. Castro Neto, Science 353 (2016) 461.

[26] A. Falin, Q. Cai, E.J.G. Santos, D. Scullion, D. Qian, R. Zhang, Z. Yang, S. Huang, K. Watanabe, T. Taniguchi, M.R. Barnett, Y. Chen, R.S. Ruoff, L.H. Li, Nat. Commun. 8 (2017) 15815.

[27] W.G. Cullen, M. Yamamoto, K.M. Burson, J.H. Chen, C. Jang, L. Li, M.S. Fuhrer, E. D. Williams, Phys. Rev. Lett. 105 (2010) 215504. 